\title{
Candidatus Rickettsia xinyangensis as Cause of Spotted Fever Group Rickettsiosis, Xinyang, China, 2015
}

\author{
Hao Li, ${ }^{1}$ Xiao-Mei Li, ${ }^{1}$ Juan Du, Xiao-Ai Zhang, Ning Cui, Zhen-Dong Yang, \\ Xiao-Jia Xue, Pan-He Zhang, Wu-Chun Cao, Wei Liu
}

In 2015, we evaluated 221 patients with undifferentiated fever and tick bite or animal exposure in Xinyang, China, for Rickettsia infection. Three with mild disease were infected with Candidatus R. xinyangensis, which clustered with $R$. fournieri and $R$. vini in phylogenetic analyses. Field investigations suggest Haemaphysalis longicornis ticks might be involved in transmission.

Spotted fever group (SFG) rickettsiae (SFGR) are obligate intracellular bacteria of the genus Rickettsia and family Rickettsiaceae and comprise $>20$ species identified as human pathogens (1). Most SFGR are transmitted by ticks (1), and flea-transmitted $R$. felis and mite-transmitted R. akari are recognized as members of the transitional group rickettsiae (2). In China, 4 different species and 1 new genotype of SFGR have been identified in association with human diseases $(3,4)$.

Clinical symptoms of SFG rickettsioses are often simply fever and rash, although several other features, such as eschar and lymphadenopathy, are also commonly described (1). Diverse manifestations of diseases can make their clinical diagnoses rather difficult. Moreover, with the aid of molecular techniques, many new pathogenic SFGR are being discovered globally with increasing frequency. This increased discovery calls for researchers to intensify their efforts investigating patients with undifferentiated febrile illness. Here, we report a case series of 3 patients in China infected with the same novel SFG Rickettsia.

Author affiliations: State Key Laboratory of Pathogen and Biosecurity, Beijing Institute of Microbiology and Epidemiology, Beijing, China (H. Li, J. Du, X.-A. Zhang, P.-H. Zhang,

W.-C. Cao, W. Liu); ShanDong First Medical University and ShanDong Academy of Medical Sciences, Tai'an, China (X.-M. Li, X.-J. Xue); People's Liberation Army 154 Hospital, Xinyang, China (N. Cui, Z.-D. Yang); Beijing Key Laboratory of Vector Borne and Natural Focus Infectious Diseases, Beijing (W. Liu)

DOI: https://doi.org/10.3201/eid2605.170294

\section{The Study}

During March-November 2015, we recruited 221 patients with undifferentiated febrile illness and history of tick bite or animal contact within the past month to a study conducted at the People's Liberation Army 154 Hospital in Xinyang, Henan Province, China. We excluded patients with severe fever with thrombocytopenia syndrome virus infection (Appendix, https://wwwnc.cdc.gov/EID/ article/26/5/17-0294-App1.pdf) and then tested for infection with SFGR.

We collected peripheral blood samples (using EDTA tubes) from patients at hospital admission and extracted DNA using the QIAmp DNA Blood Mini Kit (QIAGEN, https://www.qiagen.com). We concurrently performed nested PCRs specific for the conserved citrate synthase gene (gltA) and SFGRrestricted outer membrane protein A gene (ompA) (Appendix) (4). We then purified samples positive for amplicons and sequenced in both directions.

Three patients were found to be infected with a novel SFGR genotype with identical gltA and ompA gene sequences, which we designated Rickettsia sp. XY118. The gltA of XY118 (GenBank accession no. KU853023) had $99.6 \%(1,088 / 1,092)$ similarity with that of $R$. vini (accession no. KJ626330) and 99.6\% $(1,145 / 1,150)$ similarity with that of $R$. heilongjiangesis (accession no. CP002912) and R. fournieri (accession no. KF666471). The ompA gene sequence of XY118 (accession no. KU853021) was identical to those of undetermined Rickettsia species from ticks in China (accession no. AF169629) and Japan (accession no. AB516963) and rodents in South Korea (accession no. DQ402485). Moreover, the sequence of the ompA gene obtained in our patients had 96.1\% (299/311) similarity with the corresponding gene in $R$. vini (accession no. KX159442) and 96.5\% (335/347) similarity with that of R. fournieri (accession no. KF666477).

${ }^{1}$ These first authors contributed equally to this work. 
We collected serum samples from patients during the acute and convalescent phases of illness and tested for IgG against $R$. rickettsii by using an indirect immunofluorescence assay (Rickettsia IFA IgG; Focus Diagnostics, https://www.focusdx.com). Results showed that 2 patients had seroconverted and 1 had a 4-fold increased IgG titer (Appendix Table 2). In addition, we tested patients for acute infection with Anaplasma phagocytophilum, Ehrlichia chaffeensis, Borrelia burgdorferi, and Babesia microti by PCR and indirect immunofluorescence assay (5), and all blood samples were negative for both DNA of and specific IgG against these pathogens.

Two of 3 patients had reported history of tick bite, and 1 had reported animal contact (Table). All 3 patients had fever, asthenia, and anorexia. Two patients had eschar, 1 had lymphadenopathy, and none had rash. None of the 3 patients had any severe complications (i.e., hemorrhagic or neurologic signs or symptoms). Laboratory test results showed that 3 patients had leukopenia; 2 had thrombocytopenia; and 1 had elevated levels of hepatic aminotransferase, lactate dehydrogenase, and creatine kinase when admitted to the hospital (Appendix Figure 3). Clinical signs resolved and laboratory test findings were null (except for 1 patient with elevated hepatic aminotransferase levels) after 4-9 days' hospitalization.

To identify local natural foci of SFGR, we performed a field investigation for infections among ticks captured around the 3 patients' residences. We collected 232 hostseeking Haemaphysalis longicornis ticks and subjected each tick separately to DNA extraction with the DNeasy

\begin{tabular}{|c|c|c|c|}
\hline \multirow[b]{2}{*}{ Characteristics } & \multicolumn{3}{|c|}{ Patient no. } \\
\hline & 1 & 2 & 3 \\
\hline Age, y & 42 & 63 & 24 \\
\hline Sex & $\mathrm{F}$ & M & $\mathrm{M}$ \\
\hline History of tick bite & Yes & No & Yes \\
\hline $\begin{array}{l}\text { Time from tick bite to disease } \\
\text { onset, d }\end{array}$ & 7 & NA & 7 \\
\hline $\begin{array}{l}\text { Time from disease onset to } \\
\text { hospital admission, d }\end{array}$ & 3 & 4 & 5 \\
\hline No. days hospitalization & 4 & 9 & 5 \\
\hline \multicolumn{4}{|l|}{ Signs and symptoms } \\
\hline Fever & Yes & Yes & Yes \\
\hline Highest temperature, ${ }^{\circ} \mathrm{C}$ & 38.6 & 38.9 & 39.0 \\
\hline Dizziness & No & Yes & No \\
\hline Asthenia & Yes & Yes & Yes \\
\hline Myalgia & No & No & Yes \\
\hline Eschar & Yes & No & Yes \\
\hline Lymphadenopathy & No & No & Yes \\
\hline Anorexia & Yes & Yes & Yes \\
\hline Nausea & No & Yes & No \\
\hline Cough & No & Yes & No \\
\hline Rash & No & No & No \\
\hline
\end{tabular}

Blood \& Tissue Kit (QIAGEN). Rickettsia sp. XY118 was detected in $2(0.9 \%)$ ticks, and the nucleotide sequences of the gltA (GenBank accession no. KY617774) and ompA (accession no. KY617775) genes from these ticks were identical to those found in our patients. To further describe the genetic characteristics of this new genotype, we amplified the 16S rRNA gene ( $r r s$; accession no. KY617772), 120-kDa genus common antigen gene (ompB; accession no. KY617776), PS120 protein-encoding gene (sca4; accession no. KY617777), and 17-kDa antigen gene ( $h t r \mathrm{~A}$; accession no. KY617773). The nucleotide sequence $(1,320 \mathrm{bp})$ of $r r s$ of XY118 had $99.7 \%$ $(1,316 / 1,320)$ similarity with that of $R$. japonica (accession no. AP017602) and $99.6 \%(1,315 / 1,320)$ similarity with that of $R$. heilongiiangesis (accession no. CP002912; Appendix Figure 1). The nucleotide sequences of $h t r A$ (99.5\%), gltA (99.6\%), ompA (96.1\%), and ompB (99.8\%) from XY118 had the highest identity with the corresponding genes from $R$. vini. Compared with the partial sca4 sequence of $R$. fournieri, the corresponding sequence of XY118 contained 5 variable base pair sites and an 18-bp deletion (Appendix Figure 2). A phylogenetic tree that we constructed using the 2,546-bp nucleotide sequence of these 5 genes concatenated showed that Rickettsia sp. XY118, R. fournieri, and $R$. vini comprise a separate cluster that appears most closely related to $R$. japonica and R. heilongjiangensis (Figure). According to the gene sequence-based criteria for taxonomic classification of new Rickettsia isolates $(6,7)$, a Candidatus status could be assigned to XY118, so we named this species Candidatus Rickettsia xinyangensis.

\section{Conclusions}

We found a novel SFG Rickettsia in human patients and ticks in China and propose the name Candidatus R. xinyangensis for this species. Our phylogenetic analyses involving comparisons with 5 different rickettsial genes showed that this newly identified SFG Rickettsia was most closely related to $R$. fournieri, a strain first isolated from Argas lagenoplastis ticks in Australia in 2013 (8) that has unknown pathogenicity in humans.

Our finding of Candidatus R. xinyangensis in $0.9 \%$ of $H$. longicornis ticks suggests a natural foci of this bacterium in Xinyang. However, extended field surveys and tick surveillance are required to understand the distribution of this agent and to identify specific tick vectors.

For Candidatus R. xinyangensis, a causal relationship between infection and clinical disease may be inferred by the serologic evidence, although only 3 patients infected with this pathogen have been reported. On the other hand, considering that isolates with identical (311-bp) SFGR-restricted ompA gene sequences have been detected in $H$. yeni and $H$. longicornis ticks 


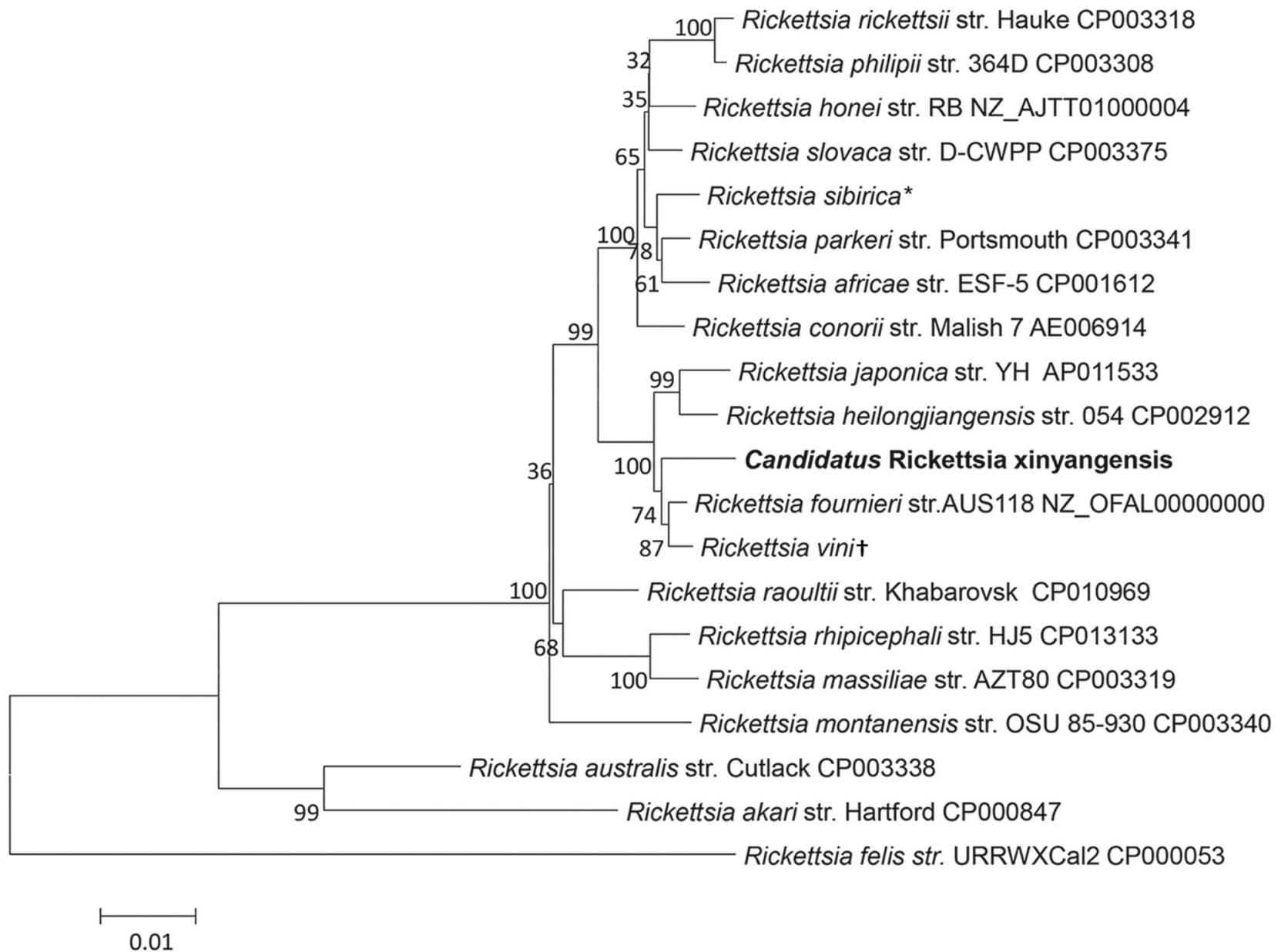

Figure. Phylogenetic analysis of concatenated nucleotide sequence of novel spotted fever group Rickettsia, Candidatus R. xinyangensis (bold), Xinyang, China, 2015. The partial nucleotide sequences of genes htrA (421 bp), gltA (1,092 bp), ompA (332 bp), ompB (456 bp), and sca4 (245 bp) were concatenated and compared via the maximum-likelihood method by using the best substitution model found (i.e., Tamura 3-parameter plus gamma) and MEGA version 5.0 (http://www.megasoftware.net). A bootstrap analysis of 1,000 replicates was applied to assess the reliability of the reconstructed phylogenies, and bootstrap values are indicated at branch nodes. Scale bar indicates the number of substitutions per nucleotide position. Str., strain. *Sequence includes the htrA gene from $R$. sibirica str. 246 (GenBank accession no. AABW01000001) and gltA (accession no. HM050296), ompA (accession no. HM050272), ompB (accession no. HM050273), and sca4 (accession no. HM050295) genes from str. RH05. †Sequence includes the htrA (accession no. KT187396), gltA (accession no. KT187394), and ompA (accession no. KT326194) genes from R. vini str. Breclav; ompB from str. 4GA09_32 (accession no. JF758826); and sca4 from str. IA-CR (accession no. KX159443).

in China $(9,10), H$. longicornis ticks in Japan (11), $H$. bispinosa ticks in Bangladesh (12), and Apodemus agarius rodents in Korea (13), Candidatus R. xinyangensis could be a tickborne infection of immense clinical relevance in humans.

In our study, patients with Candidatus R. xinyangensis infection had similar relatively mild febrile illnesses, as well as leukopenia and elevated hepatic enzyme levels, both of which are features of other SFG rickettsioses. In contrast, unlike patients with many other SFG rickettsioses, including Rocky Mountain spotted fever, our patients had eschars, not rashes $(1,14)$. However, the patients described in this report were few in number and from a single hospital, and the true disease presentation of Candidatus R. xinyangensis infection might be more variable. Future investigations to further assess the disease spectrum of this pathogen and its contribution to clinical cases are needed.

This study was supported by the China Mega-Project for Infectious Diseases (grant nos. 2018ZX10713002, 2018ZX10101003, 2018ZX10301401), the National Natural Science Foundation of China $(81825019,81472005$, and 81621005), the New Star Plan of Science and Technology of Beijing (Z171100001117089), and the Shandong Natural Science Foundation (ZR2016HL46). 


\section{About the Author}

$\mathrm{Dr}$. Li is a scientist in the State Key Laboratory of Pathogen and Biosecurity, Beijing Institute of Microbiology and Epidemiology, Beijing, China. His research interests include microbiology, epidemiology, and ecology of tickborne diseases.

\section{References}

1. Parola P, Paddock CD, Socolovschi C, Labruna MB, Mediannikov O, Kernif T, et al. Update on tick-borne rickettsioses around the world: a geographic approach. Clin Microbiol Rev. 2013;26:657-702. http:/ / dx.doi.org/10.1128/ CMR.00032-13

2. Pérez-Osorio CE, Zavala-Velázquez JE, Arias León JJ, Zavala-Castro JE. Rickettsia felis as emergent global threat for humans. Emerg Infect Dis. 2008;14:1019-23. http:/ / dx.doi.org/10.3201/eid1407.071656

3. Fang LQ, Liu K, Li XL, Liang S, Yang Y, Yao HW, et al. Emerging tick-borne infections in mainland China: an increasing public health threat. Lancet Infect Dis. 2015;15:1467-79. http://dx.doi.org/10.1016/ S1473-3099(15)00177-2

4. Li H, Cui XM, Cui N, Yang ZD, Hu JG, Fan YD, et al. Human infection with novel spotted fever group Rickettsia genotype, China, 2015. Emerg Infect Dis. 2016;22:2153-6. http:/ / dx.doi.org/10.3201/eid2212.160962

5. Liu W, Li H, Lu QB, Cui N, Yang ZD, Hu JG, et al. Candidatus Rickettsia tarasevichiae infection in eastern central China: a case series. Ann Intern Med. 2016;164:641-8. http://dx.doi.org/10.7326/M15-2572

6. Fournier PE, Dumler JS, Greub G, Zhang J, Wu Y, Raoult D. Gene sequence-based criteria for identification of new Rickettsia isolates and description of Rickettsia heilongjiangensis sp. nov. J Clin Microbiol. 2003;41:5456-65. http:/ / dx.doi.org/ 10.1128/JCM.41.12.5456-5465.2003

7. Raoult D, Fournier PE, Eremeeva M, Graves S, Kelly PJ, Oteo JA, et al. Naming of rickettsiae and rickettsial diseases. Ann N Y Acad Sci. 2005;1063:1-12. http:/ / dx.doi.org/10.1196/annals.1355.002

8. Diop A, Barker SC, Eberhard M, Barker D, Nguyen TT, Di Pinto F, et al. Rickettsia fournieri sp. nov., a novel spotted fever group rickettsia from Argas lagenoplastis ticks in Australia. Int J Syst Evol Microbiol. 2018;68:3781-4. http://dx.doi.org/10.1099/ijsem.0.003057

9. Zhang PH, Cao WC, Zhang XT, Dai XH, Chen ZG, Xu RM. Detection and identification of a new spotted fever group rickettsiae in Fujian Province of China. Chin J Pub Health. 2000;16:518-20.

10. Zou Y, Wang Q, Fu Z, Liu P, Jin H, Yang H, et al. Detection of spotted fever group Rickettsia in Haemaphysalis longicornis from Hebei Province, China. J Parasitol. 2011;97:960-2. http:/ / dx.doi.org/10.1645/GE-2751.1

11. Ishikura M, Ando S, Shinagawa $Y$, Matsuura $K$, Hasegawa S, Nakayama T, et al. Phylogenetic analysis of spotted fever group rickettsiae based on gltA, 17-kDa, and rOmpA genes amplified by nested PCR from ticks in Japan. Microbiol Immunol. 2003;47:823-32. http://dx.doi.org/10.1111/j.1348-0421.2003.tb03448.x

12. Qiu Y, Nakao R, Thu MJ, Akter S, Alam MZ, Kato S, et al. Molecular evidence of spotted fever group rickettsiae and Anaplasmataceae from ticks and stray dogs in Bangladesh. Parasitol Res. 2016;115:949-55. http:/ /dx.doi.org/10.1007/ s00436-015-4819-y

13. Kim CM, Yi YH, Yu DH, Lee MJ, Cho MR, Desai AR, et al. Tick-borne rickettsial pathogens in ticks and small mammals in Korea. Appl Environ Microbiol. 2006;72:5766-76. http://dx.doi.org/10.1128/AEM.00431-06

14. Dantas-Torres F. Rocky Mountain spotted fever. Lancet Infect Dis. 2007;7:724-32. http://dx.doi.org/10.1016/ S1473-3099(07)70261-X

Address for correspondence: Wei Liu or Hao Li, State Key Laboratory of Pathogen and Biosecurity, Beijing Institute of Microbiology and Epidemiology, 20 Dong-Da St, Fengtai District, Beijing 100071, China; email: liuwei@bmi.ac.cn or lihao_1986@126.com 